DOI: $10.15593 / 2224-9982 / 2017.50 .03$

УДК 629.7.036.34; 678(075)

\author{
Н.А. Кошелева, Г.С. Шипунов, А.А. Воронков, \\ Н.П. Меркушева, А.А. Тихонова
}

Пермский национальный исследовательский политехнический университет, Пермь, Россия

\author{
ЭКСПЕРИМЕНТАЛЬНЫЕ ИССЛЕДОВАНИЯ ПО ОПРЕДЕЛЕНИЮ \\ ДЕФОРМАЦИЙ ОБРАЗЦОВ ИЗ ПОЛИМЕРНОГО КОМПОЗИЦИОННОГО \\ МАТЕРИАЛА С ПРИМЕНЕНИЕМ ВОЛОКОННО-ОПТИЧЕСКИХ ДАТЧИКОВ
}

\begin{abstract}
Рассматриваются результаты экспериментальных исследований по определению деформаций при неоднородном напряженно-деформированном состоянии и модуля упругости композиционного материала при испытаниях на растяжение и сжатие с применением встроенных в материал волоконно-оптических датчиков деформаций (ВОДД) на брэгговских решетках. Рассмотрены образцы из стеклопластика с внедренными оптоволоконными линиями в форме прямоугольной пластины и с V-образным вырезом. В пластине с вырезами достигается градиентное поле деформаций, рассматривается возможность ВОДД фиксировать данное поле. Результаты измерения градиентных полей деформаций в пластине с вырезами сравниваются с результатами, полученными при использовании трехмерной цифровой оптической системы Vic3D, и результатами численных расчетов на основе метода конечных элементов. Демонстрируется, что рассмотренные три варианта получения информации о деформациях различаются в пределах 5 \%. Результаты экспериментов при одноосном растяжении и сжатии демонстрируют возможность определения модуля упругости с помощью ВОДД. Приведено сравнение экспериментальных результатов по определению модуля упругости, получаемого с применением стандартного испытательного оборудования, видеосистемы контроля напряженно-деформированного состояния Vic3D и оптоволоконных датчиков на брэгговской решетке.

Ключевые слова: полимерный композиционный материал, стеклопластик, волоконно-оптические датчики, оптоволокно, брэгговская решетка.
\end{abstract}

\author{
N.A. Kosheleva, G.S. Shipunov, A.A. Voronkov, \\ N.P. Merkusheva, A.A. Tihonova \\ Perm National Research Polytechnic University, Perm, Russian Federation

\section{DETERMINATION OF THE STRESS-STRAIN STATE FIELDS OF SAMPLES FROM A POLYMERIC COMPOSITE MATERIAL USING FIBER OPTIC SENSORS}

This work presents the results of the experimental studies of strain distribution in the composite specimens using optical sensors based on fiber Bragg gratings (FBG) embedded into the material. Both flat bar and $V$-notched specimens were taken for tensile tests which were carried out on Instron 5982 machine. Also, the optical system Vic3D was used for independent registration of strain fields on the specimen surface. The results of tensile testing of flat specimens showed the general possibility of uniform strain measurement and were used for calculation of calibration factor for the next following tests. A series of repeated uniaxial tension and compression tests was performed on flat bar samples to determine the material modulus of elasticity using FBG sensors. The tension of V-notched specimen leads to occurrence of non-uniform fields of strain. The results of the strain gradient registration in the V-notched composite specimen using FBG sensors were compared with the digital images received from the optical system Vic3D. The difference between the results of strain measurement obtained with the different approaches did not exceed $5 \%$.

Keywords: polymeric composite material, fiberglass, optical fiber sensors, fiber, Bragg grating. 


\section{Введение}

Одним из основных вопросов при создании перспективных изделий для авиационной и ракетно-космической техники из полимерных композиционных материалов является обеспечение надежности при длительной эксплуатации. Особенно остро данный вопрос стоит при использовании композитов в гражданском авиастроении и двигателестроении. При этом по своей природе композиционный материал является более сложным объектом для изучения механических свойств. Он обладает анизотропией, существенной зависимостью свойств от температуры и технологии изготовления. В ряде случаев свойства материала в составе конструкции вообще невозможно определить на стандартных образцах, а влияние технологических факторов, таких как характеристики сырья и компонентов материала, параметров технологических процессов, с трудом возможно спрогнозировать. Однако в самом принципе создания композиционного материала и конструкции одновременно из ряда отдельных компонентов, фактически создания материала-детали, можно найти решение проблемы обеспечения его надежности $[1,2]$. Это создание материала с возможностью диагностики и мониторингом своего состояния в процессе стендовых, летных испытаний и, в перспективе, при длительном процессе эксплуатации с помощью волоконно-оптических датчиков деформации [3, 4].

Волоконно-оптические датчики могут измерять различные физические механические величины, способны выдерживать деформации, сопоставимые с деформациями композита, невосприимчивы к электрическим помехам и в жестких условиях имеют преимущества, в том числе по чувствительности. Одна из главных задач при использовании оптоволоконных датчиков в композиционных материалах связана с измерением деформации. В России большой опыт работы с волоконно-оптическими датчиками на основе брэгговских решеток имеется в ФГУП «ВИАМ» [5]. В своей работе они рассмотрели возможные проблемы при измерении деформации конструкций из композиционных материалов, также затронуты вопросы влияния интегрированного оптоволокна на свойства полимерного композиционного материала, ввода-вывода оптоволокна из композита, выбора модели, связывающей деформацию оптического волокна с измерением ее резонансной длины волны, проблема калибровки.

Мировой опыт использования волоконно-оптических датчиков рассматривается в достаточно большом количестве работ. Есть отдельные публикации, посвященные методам и средствам измерения [6-8]. Рассматриваются вопросы влияния внедренных оптических волокон на физико-механические свойства композиционного материала [9]. В работе [10] анализируются возможности выполнения волоконно-оптическими датчиками нескольких функций. Возможности волоконно-оптических датчиков для анализа дефектов между слоями композита приводятся в работе [11]. Примером эффективного использования волоконно-оптических датчиков в практических приложениях является работа [12], где описывается применение датчиков для мониторинга стекловолоконных композиционных секций автомобильного моста. Реализация рассмотренных идей в реальных конструкциях рассмотрена в работе [13], где дается подробное описание технологии использования встроенных волоконно-оптических датчиков для регистрации деформации композиционного материала с результатами эксперимента по регистрации деформации в пластине при ее четырехточечном изгибе.

\section{Подготовка к проведению экспериментальных исследований}

Для проведения экспериментальных исследований по оценке способности волоконнооптических датчиков фиксировать деформации в неоднородном поле напряжений были изготовлены образцы композиционного материала из препрега стеклопластика ВПС-48 в форме прямоугольной пластины и с $\mathrm{V}$-образным вырезом, со встроенным оптоволокном, на котором расположены несколько ВОДД (рис. $1, a$ ). Изготовляли в соответствии с технологическими рекомендациями производителя материала: раскрой армирующего материала на плоттере Zund G3 L-2500; выкладка на технологическую оснастку 20 слоев препрега; укладка оптоволоконных 
датчиков между 10-м и 11-м слоями армирующего материала в соответствии со схемой, приведенной на рис. $1, \sigma$; сборка технологического пакета для проведения подпрессовки материала; технологическое обеспечение защиты выходов оптоволокна от температуры и давления (рис. 2); проведение режима полимеризации; демонтаж технологического пакета.

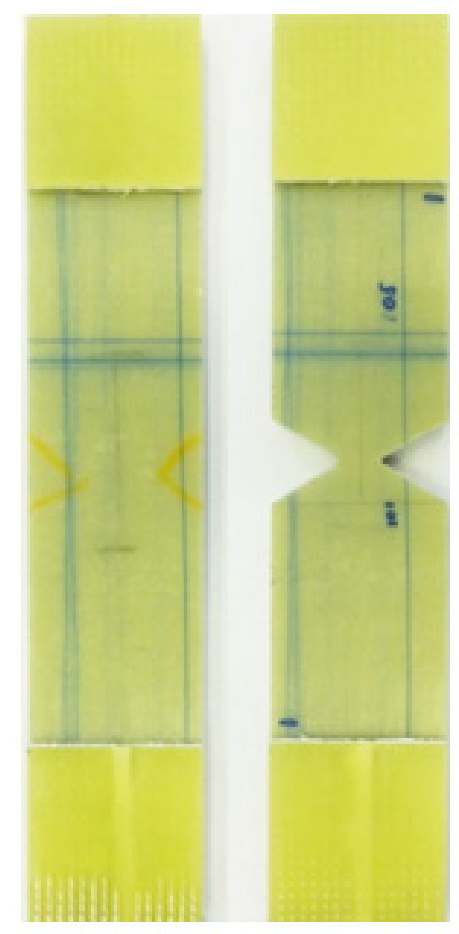

$a$

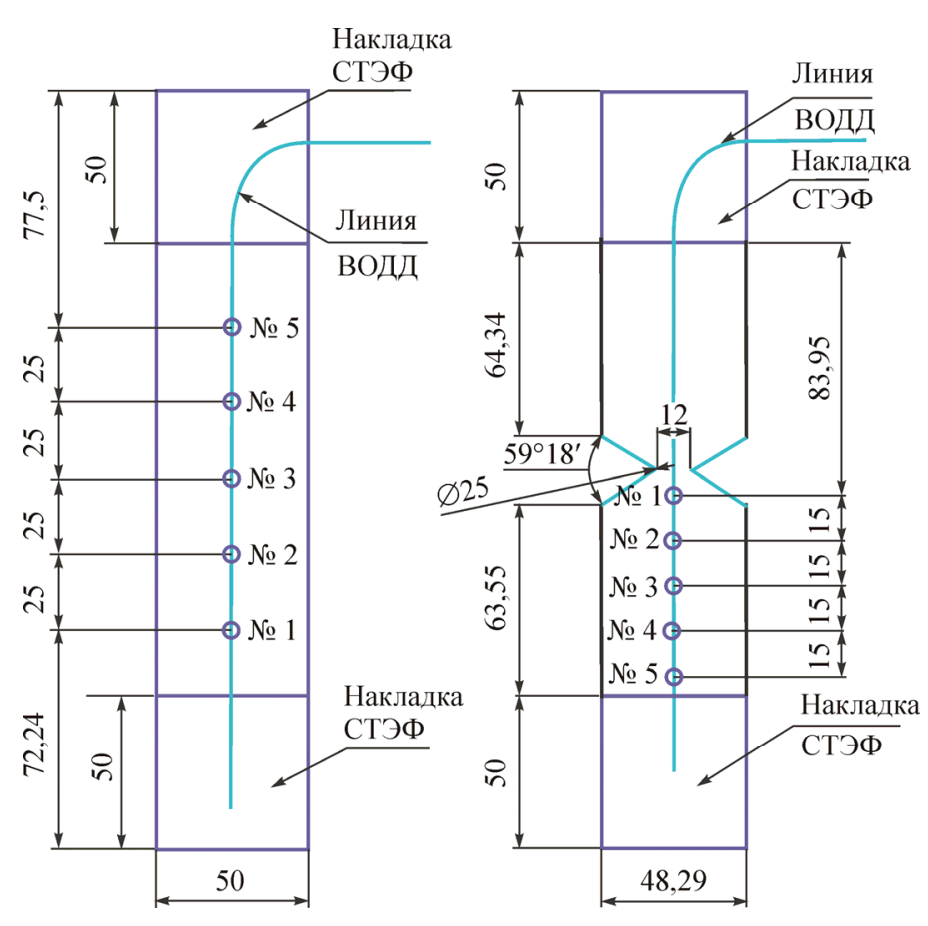

$\sigma$

Рис. 1. Образцы стеклопластика с внедренными ВОДД (a), схемы расположения линии ВОДД в образцах (б)

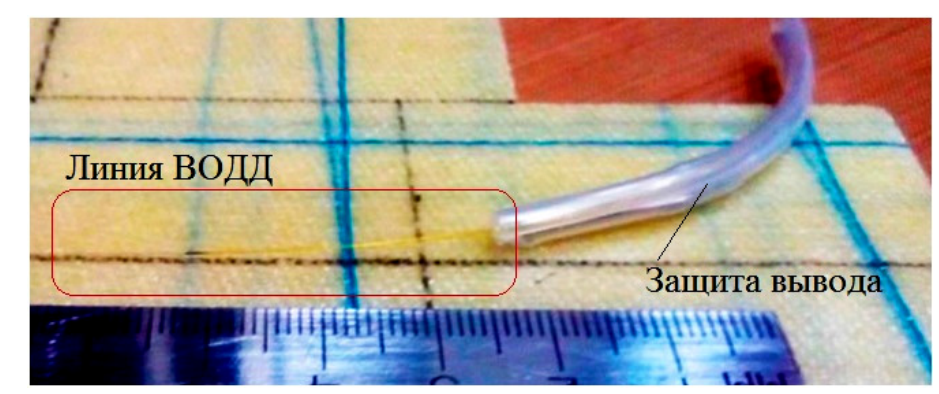

Рис. 2. Укладка линий ВОДД и защита выводов

Процесс полимеризации и отверждения композиционного материала с внедренными ВОДД производился в пресce Langzauner с непрерывным контролем температуры и давления, а также снимались показания с внедренных ВОДД с использованием интеррогатора ASTRO X327.

На образцах в форме прямоугольной пластины был проведен технологический эксперимент по калибровке волоконно-оптических датчиков, внедренных в ПКМ. Второй тип образцов c $V$-образным вырезом использовался для экспериментов по измерению неоднородных полей деформаций. При этом измерение деформаций осуществлялось с учетом результатов калибровочных экспериментов на первой серии образцов. В дальнейшем на образцах без выреза проведена серия экспериментов по определению модуля упругости материала при одноосном растяжении и сжатии с применением волоконно-оптических датчиков. 
Структурная схема измерения одноосной деформации в плоском образце из ПКМ с использованием внедренных ВОДД на основе брэгговской решетки представлена на рис. 3. Здесь 1 - образец; 2 - участок оптического волокна с ВОДД; 3 - интеррогатор; 4 - компьютер; 5 широкополосный спектр; 6 - отраженный спектр; 7 - вывод на компьютер. Согласно этой схеме интеррогатор генерирует и передает по оптическому волокну широкополосный оптический сигнал $\psi(\lambda)(\lambda$ - длина волны). На брэгговской решетке часть этого сигнала отражается. Основная часть отраженного оптического сигнала имеет длину волны $\lambda$ - резонансная длина волны отраженного спектра. Величина этой волны прямо пропорциональна эффективному показателю преломления $n$ и геометрической длине периода брэгговской решетки $L$ (см. рис. 3). Именно эта зависимость и определила возможность регистрации изменения относительной длины периода $\Delta L / L$ по анализу изменения относительной величины резонансной длины волны отраженного спектра $\Delta \lambda / \lambda$. Согласно работе [13], имеется зависимость

$$
\frac{\Delta \lambda}{\lambda}=K_{\varepsilon} \varepsilon+K_{T} \Delta T,
$$

где $\varepsilon$ - относительная деформация брэгговской решетки вдоль ее оси за счет механического деформирования; $\Delta T$ - изменение температуры оптического волокна в месте расположения брэгговской решетки с момента начала измерений резонансной длины волн отраженного спектра; $K_{\varepsilon}, K_{T}$ - соответственно деформационный и температурный коэффициенты; $\Delta \lambda$ - разница величин резонансных длин волн отраженного спектра соответственно в текущий $(\lambda)$ и начальный $\left(\lambda^{*}\right)$ моменты времени, $\Delta \lambda=\lambda-\lambda^{*}$.

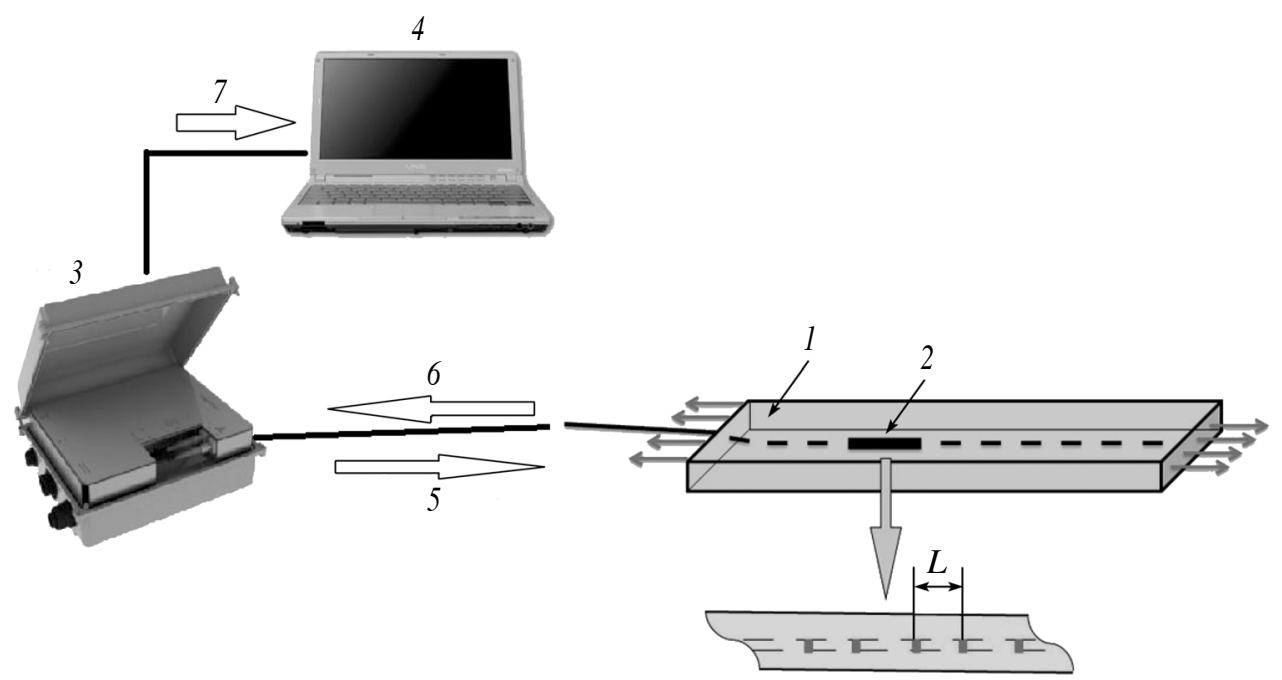

Рис. 3. Схема измерения одноосной деформации в плоском образце из ПКМ с использованием внедренных ВОДД

Для испытаний на одноосное растяжение и сжатие используется универсальная электромеханическая система Instron 5882. Независимая регистрация полей деформаций, особенно необходимая для калибровочных экспериментов, осуществлялась с помощью трехмерной цифровой оптической системы Vic3D (рис. 4) [14].

Совместно с экспериментом проводилось математическое моделирование в рамках анизотропной теории упругости для дополнительной оценки работоспособности волоконнооптических датчиков. Расчет проводился в программном пакете ANSYS с использованием метода конечно-элементного анализа. 


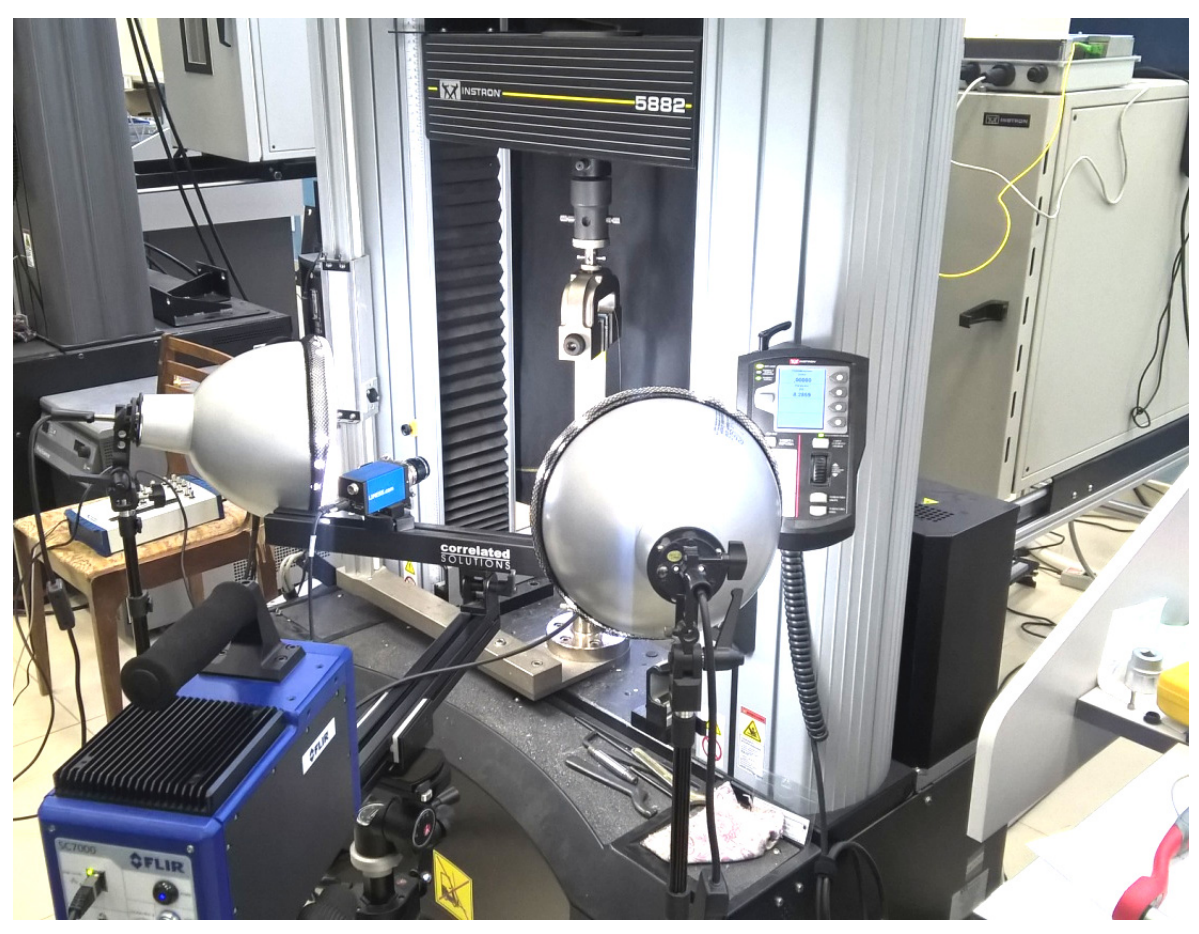

Рис. 4. Общий вид оборудования при проведении экспериментов

\section{Результаты экспериментов и их анализ}

Ha первом этапе осуществлен цикл испытаний на одноосное растяжение образцов c $V$-образным вырезом. Такая форма образца обеспечивает градиент продольной деформации вдоль осевой линии, что было определено по результатам предварительного математического моделирования. Эксперимент проходил со скоростью 2 мм/мин до нагрузки 10 кН. Дополнительно снимались значения деформаций в местах расположения ВОДД с использованием трехмерной цифровой оптической системы Vic3D, с частотой 15 кадров в секунду.

На рис. 5 представлены результаты распределения продольной деформации вдоль осевой линии образца на момент времени нагружения $t=18,9 \mathrm{c}$, полученные с использованием оптической системы (пунктирная линия) и ВОДД (круги). Результаты измерений ВОДД представлены с учетом коэффициентов калибровки, полученных по результатам испытаний образцов без концентратора. На этом же графике приведены результаты, полученные на основе математического моделирования (сплошная линия). На данном графике и для всех моментов времени нагружения в зоне концентрации деформаций отличие результатов, полученных с ВОДД, от результатов, полученных оптической измерительной системой и численным моделированием, не превышает $5 \%$.

На втором этапе экспериментальных исследований была проведена оценка модуля упругости композиционного материала при одноосном растяжении и сжатии. Испытания проводились на прямоугольном образце без концентраторов с пятью внедренными оптоволоконными датчиками. Также модуль упругости контролировался с помощью стандартной испытательной машины и видеосистемы Vic3D. 


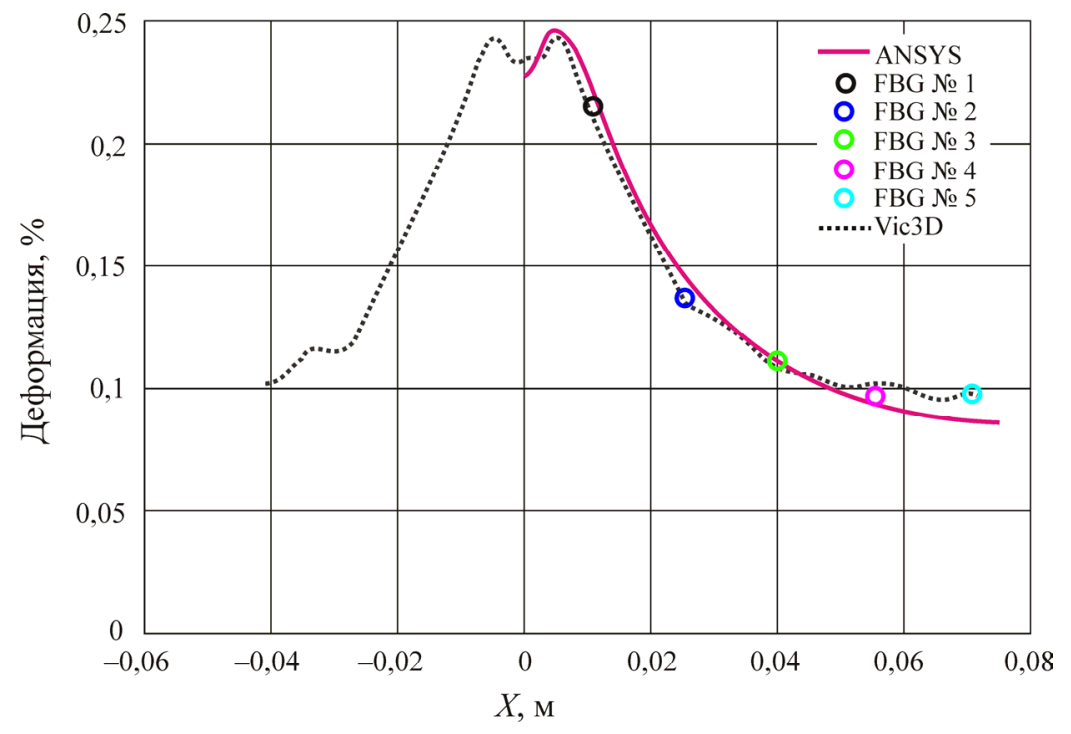

Рис. 5. Результаты испытаний на одноосное растяжение в неоднородном поле деформаций

Эксперимент проводился по следующей схеме: предварительная нагрузка до $20 \%$ от $F_{\max }$ $(20$ кН) с последующей разгрузкой до $5 \%(5$ кH), затем трехкратное нагружение/разгружение (5-30 кН), далее переход в область сжатия, предварительная нагрузка до -11 кН, с разгрузкой до -7 кН, далее трехкратное нагружение/разгружение $(-7 \ldots-14)$ кН. Значение деформаций, полученных с волоконно-оптических датчиков и видеосистемы Vic3D при проведении эксперимента, приведено на рис. 6.

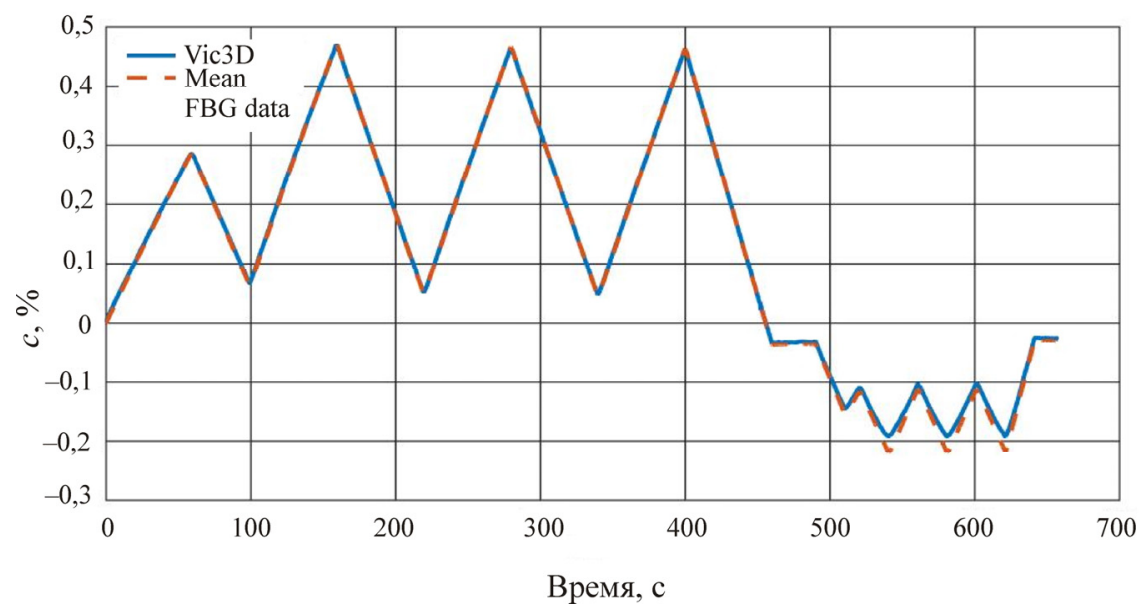

Рис. 6. Деформации образца при проведении одноосного растяжения и сжатия

При проведении экспериментальных исследований волоконно-оптические датчики достаточно корректно описывают процесс повторяющейся нагрузки в области растяжения. При рассмотрении сжимающих напряжений возникает различие в показаниях, получаемых c Vic3D и ВОДД, составляющее порядка $10 \%$. На рис. 7 представлена зависимость нагрузки, прилагаемой испытательной машиной, от деформаций. Разница в показаниях тоже составляет порядка $10 \%$. После экспериментов проведен анализ модуля упругости, получаемого при одноосном растяжении, и сжатие с применением системы Vic3D и ВОДД. Полученные результаты приведены в таблице. 


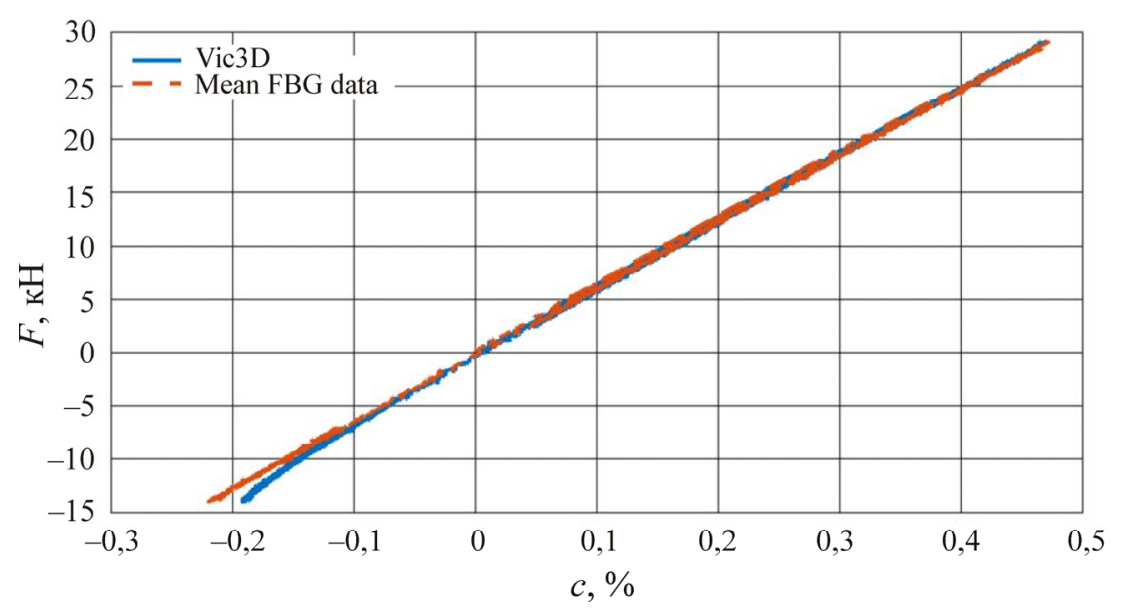

Рис. 7. Сравнение результатов, полученных с ВОДД и Vic3D

Значение модуля упругости при экспериментальных исследованиях по растяжению и сжатию

\begin{tabular}{|l|c|c|c|c|c|c|c|}
\hline \multirow{2}{*}{ Пик } & \multicolumn{7}{|c|}{ Значения модуля упругости, ГПа } \\
\cline { 2 - 7 } & Vic3D & ВОДД № 1 & ВОДД № 2 & ВОДД № 3 & ВОДД № 4 & ВОДД № 5 & Различие, \% \\
\hline \multicolumn{7}{|c|}{ Растяжение } \\
\hline Пик 1 & 25,98 & 25,35 & 25,65 & 25,47695 & 25,45 & 25,35 & 1,80 \\
\hline Пик 2 & 26,50 & 24,94 & 24,93 & 25,13318 & 24,72 & 24,94 & 5,97 \\
\hline Пик 3 & 26,68 & 25,95 & 25,94 & 26,2524 & 25,71 & 25,95 & 2,77 \\
\hline \multicolumn{7}{|c|}{ Сжатие } \\
\hline Пик 1 & 34,47 & 28,20 & 26,67 & 26,83 & 28,49 & 28,20 & 20,40 \\
\hline Пик 2 & 31,13 & 26,63 & 26,63 & 26,91 & 24,59 & 26,63 & 15,34 \\
\hline Пик 3 & 36,62 & 26,27 & 25,62 & 25,92 & 27,28 & 26,27 & 28,46 \\
\hline
\end{tabular}

Анализируя результаты, приведенные в таблице, можно сделать вывод, что при определении модуля упругости материала на стандартных образцах при одноосном растяжении различие в показаниях независимой видеосистемы Vic3D и внедренных оптоволоконных датчиков не превышает $6 \%$. При переходе в область сжатия всё не так однозначно. Различие показаний превышает $25 \%$. Это обусловлено тем, что при проведении испытаний у исследуемого образца наблюдались изгибные деформации, обусловленные его геометрическими размерами. Для более точной оценки модуля упругости необходимо провести эксперимент на стандартизированных образцах для испытаний на одноосное сжатие и уточненную оценку возможности определения модуля упругости материала с применением волоконно-оптических датчиков.

\section{Заключение}

Представлены экспериментальные результаты, демонстрирующие эффективность ВОДД для регистрации градиентных полей деформации. Достоверность этих результатов подтверждена численными расчетами и измерениями полей деформации цифровой оптической системой Vic3D. Проведена оценка возможности определения модуля упругости материала с применением встроенных волоконно-оптических датчиков. Получено совпадение результатов, снимаемых с применением независимой системы Vic3D и ВОДД, порядка $6 \%$ при испытании на одноосное растяжение. При проведении экспериментов по одноосному сжатию разница в пока- 
заниях составляет более 25 \% и обусловлена изгибными деформациями. Для уточнения данных результатов необходимо провести дополнительный комплекс экспериментальных исследований при обеспечении чистого сжатия.

Работа выполнена в Пермском начиональном исследовательском политехническом университете при финансовой поддержке Российского научного фонда ( проект №15-19-00243)

\section{Библиографический список}

1. Concise encyclopedia of composite materials (Advances in Materials Sciences and Engineering) / ed. A. Kelly. - England: Revised Edition, 1994. - 350 p.

2. Committee on new materials for advanced civil aircraft, national materials advisory board, aeronautics and space engineering board, commission on engineering and technical systems. National Research Council. New Materials for Next-Generation Commercial Transports. - Washington: National academy press, 1996. - 98 p.

3. Monitoring of fatigue damage of composite structures by using embedded intensity-based optical fiber sensors / D. Lee, J. Lee, I. Kwon, D. Seo // Smart Mater. Struct. - 2001. - Vol. 10. - P. 285-292.

4. Delamination detection in laminate composites with an embedded fiber optical interferometric sensor / C.K. Leung, Z. Yang, Y. Xu, P. Tong, S.K. Lee // Sensors and Actuators A: Phys. - 2005. - Vol. 119, № 2. P. 336-344.

5. К вопросу об интеграции оптоволокна в ПКМ и измерении деформации материала с помощью волоконных брэгговских решеток / В.В. Махсидов, М.Ю. Федотов, А.М. Шиенок, М.А. Зуев // Механика композиционных материалов и конструкций. - 2014. - Т. 20, № 4. - С. 568-574.

6. The signal characteristics of reflected spectra of fiber Bragg grating sensors with strain gradients and grating lengths / D. Kanga, S. Parkb, C. Hongb, C. Kimb // NDT\&E International. - 2005. - Vol. 38, № 8. P. 712-718.

7. Multi-axial strain transfer from laminated CFRP composites to embedded Bragg sensor: I. Parametric study / G. Luyckx, E. Voet, W. De Waele, J. Degrieck // Smart Mater. Struct. - 2010. - Vol. 19. - Art. ID 105017.

8. Mawatari T., Nelson D. A multi-parameter Bragg grating fiber optic sensor and triaxial strain measurement // Smart Mater. Struct. - 2008. - Vol. 17. - Art. ID 035033.

9. Characterization of the response of fibre Bragg grating sensors subjected to a two-dimensional strain field / I. Bosia, P. Giaccari, J. Botsis, M. Facchini, H. Limberger, R. Salathe // Smart Mater. Struct. - 2003. Vol. 12. - P. 925-934.

10. Development of smart composite structures with small-diameter ber Bragg grating sensors for damage detection: quantitative evaluation of delamination length in CFRP laminates using lamb wave sensing / N. Takeda, Y. Okabe, J. Kuwahara, S. Kojima, T. Ogisu // Compos. Sci. Technol. - 2005. - Vol. 65. - P. 25752587.

11. A novel time-division multiplexing ber Bragg grating sensor interrogator for structural health monitoring / Y. Dai, Y. Liu, J. Leng, G. Deng, A. Asundi // Opt. Lasers Eng. - 2009. - Vol. 47. - P. 1028-1033.

12. Integration and assessment of fibre Bragg grating sensors in an all-fibre reinforced polymer composite road bridge / Y. Gebremichaela, W. Lia, W. Boylea, B. Meggittb, K. Grattana, B. McKinleya, G. Fernandoc, G. Kisterc, D. Winterc, L. Canningd, S. Luke // Sensors and Actuators A. - 2005. - Vol. 118, № 1. - P. 78-85.

13. Применение оптического волокна в качестве датчиков деформации в полимерных композиционных материалах / Е.Н. Каблов, Д.В. Сиваков, И.Н. Гуляев, К.В. Сорокин, М.Ю. Федотов, Е.М. Дианов, С.А. Васильев, О.И. Медведков // Все материалы. Энциклопедический справочник. - 2010. - № 3. C. $10-15$.

\section{References}

1. Concise Encyclopedia of Composite Materials (Advances in Materials Sciences and Engineering). Ed. A. Kelly. England: Revised Edition, 1994. 350 p.

2. Committee on New Materials for Advanced Civil Aircraft, National Materials Advisory Board, Aeronautics and Space Engineering Board, Commission on Engineering and Technical Systems, National Research Council. New Materials for Next-Generation Commercial Transports. Washington: National academy press, $1996.98 \mathrm{p}$. 
3. Lee D., Lee J., Kwon I., Seo D. Monitoring of fatigue damage of composite structures by using embedded intensity-based optical fiber sensors. Smart Mater. Struct., 2001, Vol. 10, pp. 285-292.

4. Leung C.K., Yang Z., Xu Y., Tong P., Lee S.K. Delamination detection in laminate composites with an embedded fiber optical interferometric sensor. Sensors and Actuators A: Phys., 2005, Vol. 119, no. 2, pp. 336-344.

5. Mahsidov V.V., Fedotov M.YU., Shienok A.M., Zuev M.A. K voprosu ob integracii optovolokna v PKM I izmerenii deformacii materiala s pomoshch'yu volokonnyh brehggovskih reshetok [To the question of fiber integration in in composites and measurement of material deformation using fiber Bragg gratings]. Mekhanika kompozicionnyh materialov I konstrukcij, 2014, Vol. 20, no. 4, pp. 568-574.

6. Kanga D., Parkb S., Hongb C., Kimb C. The signal characteristics of reflected spectra of fiber Bragg grating sensors with strain gradients and grating lengths. NDT\&E International, 2005, Vol. 38, no. 8, pp. 712-718.

7. Luyckx G., Voet E., De Waele W., Degrieck J. Multi-axial strain transfer from laminated CFRP composites to embedded Bragg sensor: I. Parametric study. Smart Mater. Struct., 2010, Vol. 19, Art. ID 105017.

8. Mawatari T., Nelson D. A multi-parameter Bragg grating fiber optic sensor and triaxial strain measurement. Smart Mater. Struct., 2008, Vol. 17, Art. ID 035033.

9. Bosia I., Giaccari P., Botsis J., Facchini M., Limberger H., Salathe R. Characterization of the response of fibre Bragg grating sensors subjected to a two-dimensional strain field. Smart Mater. Struct., 2003, Vol. 12, pp. 925-934.

10. Takeda N., Okabe Y., Kuwahara J., Kojima S., Ogisu T. Development of smart composite structures with small-diameter ber Bragg grating sensors for damage detection: quantitative evaluation of delamination length in CFRP laminates using lamb wave sensing. Compos. Sci. Technol., 2005, Vol. 65, pp. 2575-2587.

11. Dai Y., Liu Y., Leng J., Deng G., Asundi A. A novel time-division multiplexing ber Bragg grating sensor interrogator for structural health monitoring. Opt. Lasers Eng., 2009, Vol. 47, pp. 1028-1033.

12. Gebremichaela Y., Lia W., Boylea W., Meggittb B., Grattana K., McKinleya B., Fernandoc G., Kisterc G., Winterc D., Canningd L., Luke S. Integration and assessment of fibre Bragg grating sensors in an all-fibre reinforced polymer composite road bridge. Sensors and Actuators A, 2005, Vol. 118, no. 1, pp. 78-85.

13. Kablov E.N., Sivakov D.V., Gulyaev I.N., Sorokin K.V., Fedotov M.YU., Dianov E.M., Vasil'ev S.A., Medvedkov O.I. Primenenie opticheskogo volokna $\mathrm{v}$ kachestve datchikov deformacii $\mathrm{v}$ polimernyh kompozicionnyh materialah [The use of optical fiber as strain gauges in polymer composite materials]. Vse materialy, 2010, no. 3, pp. 10-15.

\section{Об авторах}

Кошелева Наталья Александровна (Пермь, Россия) - кандидат технических наук, научный сотрудник Научно-исследовательской лаборатории интеллектуальных материалов и конструкций ФГБОУ ВО ПНИПУ (614990, г. Пермь, Комсомольский пр., д. 29, e-mail: nataly.kosheleva@gmail.com).

Шипунов Глеб Сергеевич (Пермь, Россия) - кандидат технических наук, научный сотрудник Научно-образовательного центра акустических исследований, разработки и производства композитных и звукопоглощающих авиационных конструкций ФГБОУ ВО ПНИПУ (614990, г. Пермь, Комсомольский пр., д. 29, e-mail: shipunov-kt@ pstu.ru).

Воронков Андрей Александрович (Пермь, Россия) - младший научный сотрудник Научнообразовательного центра акустических исследований, разработки и производства композитных и звукопоглощающих авиационных конструкций ФГБОУ ВО ПНИПУ (614990, г. Пермь, Комсомольский пр., д. 29, e-mail: AAVoronkov714@gmail.com).

Меркушева Наталья Павловна (Пермь, Россия) - ведущий инженер Научно-образовательного центра акустических исследований, разработки и производства композитных и звукопоглощающих авиационных конструкций ФГБОУ ВО ПНИПУ (614990, г. Пермь, Комсомольский пр., д. 29, e-mail: natalya.merkusheva@mail.ru).

Тихонова Александра Алексеевна (Пермь, Россия) - лаборант Научно-образовательного центра акустических исследований, разработки и производства композитных и звукопоглощающих авиационных конструкций ФГБОУ ВО ПНИПУ (614990, г. Пермь, Комсомольский пр., д. 29, e-mail: tikaleksandra@yandex.ru). 


\begin{abstract}
About the authors
Nataly A. Kosheleva (Perm, Russian Federation) - Ph. D. in Technical Sciences, Researcher, Laboratory of Materials and Structures with Embedded Intellegence, Perm National Research Polytechnic University (29, Komsomolski av.,Perm, 614990, Russian Federation, e-mail: nataly.kosheleva@gmail.com).

Gleb S. Shipunov (Perm, Russian Federation) - Ph. D. in Technical Sciences, Researcher, Scientific \& Educational Center for Aviation Composite Technologies, Perm National Research Polytechnic University (29, Komsomolski av., Perm, 614990, Russian Federation, e-mail: shipunov-kt@ pstu.ru).

Andrey A. Voronkov (Perm, Russian Federation) - Junior Researcher, Scientific \& Educational Center for Aviation Composite Technologies, Perm National Research Polytechnic University (29, Komsomolski av., Perm, 614990, Russian Federation, e-mail: AAVoronkov714@gmail.com).

Nataly P. Merkusheva (Perm, Russian Federation) - Leading Engineer, Scientific \& Educational Center for Aviation Composite Technologies, Perm National Research Polytechnic University (29, Komsomolski av., Perm, 614990, Russian Federation, e-mail: natalya.merkusheva@mail.ru).

Aleksandra A. Tihonova (Perm, Russian Federation) - Laboratory Assistant, Scientific \& Educational Center for Aviation Composite Technologies, Perm National Research Polytechnic University (29, Komsomolski av., Perm, 614990, Russian Federation, e-mail: tikaleksandra@ yandex.ru).
\end{abstract}

Получено 12.07.2017 